\title{
TIME FOR REASSESSMENT: A REVIEW OF BETA-BLOCKERS IN the Setting of Cocaine Associated Chest Pain and Acute CORONARY SYNDROME
}

Jonathan Finkel MD and Gregary Marhefka, MD

\section{Introduction}

Cocaine is the second most commonly used illicit drug in the United States, ${ }^{1}$ and is the most frequent illicit substance to precipitate an emergency room visit, responsible for over 550,000 visits in 2007 alone. ${ }^{2}$ The majority of patients present with a chief complaint of chest pain ${ }^{3}$, and approximately $6 \%$ are diagnosed with cocaine associated myocardial infarction. ${ }^{4}$ For decades it has been thought that beta-blockade in the setting of cocaine use would precipitate coronary vasospasm and worsen cardiovascular outcomes due to unopposed alpha receptor stimulation. In 1999 this thinking was incorporated into the ACC/AHA guidelines, which currently recommend beta-blockers in all patients with an acute coronary syndrome except in the setting of prior cocaine use. ${ }^{4}$ Recently there have been several studies suggesting benefit from beta-blocker administration in patients with cocaine associated chest pain and myocardial ischemia.

Given the recent data that call into question the validity of avoiding beta-blockers in patients with recent cocaine use, the goal of this article is to critically review the body of evidence that exists on beta-blocker usage in the setting of cocaine induced chest pain and myocardial ischemia.

Based on our findings it is clear that the current recommendations and theories need to be scrutinized and that additional outcomes based, randomized prospective human trials be conducted to effectively examine the possible benefits of beta-blocker usage in these patients.

\section{Methods}

We performed a comprehensive search of the medical literature concerning beta-blocker usage in the setting of cocaine intoxication, and cocaine associated chest pain and myocardial ischemia. The literature search was conducted using PUB Med and included English language publications from 1960-2010.

Keywords: Cocaine, beta-blockers, cocaine chest pain, MI, emergency department.

\section{Current ACC/AHA Guidelines For Beta-blockers in Acute Coronary Syndrome}

Beta-blockers have been shown by multiple large randomized trials to decrease mortality in patients presenting with cardiac ischemia and ST-elevation myocardial infarction (STEMI). In the ISIS- 1 trial, more than 16,000 patients with suspected acute MI were enrolled within 12 hours of onset of symptoms. Subjects receiving atenolol therapy showed a reduction in 7-day mortality from $4.3 \%$ to $3.7 \%$ ( $\mathrm{p}<0.02) .{ }^{7}$ In the Metoprolol In Acute Myocardial Infarction (MIAMI) trial, more than 5700 subjects with evolving MI were randomly assigned to receive placebo or metoprolol. Fifteen-day mortality was reduced with metoprolol from $4.9 \%$ to $4.3 \%$. $^{9}$ These trials form the basis of the ACC/AHA guidelines, which recommend beta-blocker therapy in all patients with STEMI ${ }^{5}$, as well as NSTEMI/Unstable angina ${ }^{6}$, as long as there are no contraindications.

\section{Beta-blocker Usage in the Setting of Cocaine Associated Chest Pain/ACS}

The guidelines go on to state that "Beta-blockers should not be administered to patients with STEMI precipitated by cocaine use because of the risk of exacerbating coronary spasm," ${ }^{5}$ citing a review article by Kloner et al. ${ }^{8}$ published in Circulation in 1993. This article discusses the possibility of coronary vasoconstriction following beta-blocker administration in the setting of recent cocaine use, citing studies by Shannon et al. ${ }^{10}$ and Lange et al. ${ }^{11}$

Shannon et al., ${ }^{10}$ administered cocaine followed by a beta-blocker to ten canine subjects while continually monitoring systemic and coronary hemodynamics. This study found that beta blockade in the setting of cocaine intoxication worsened coronary vasoconstriction, and decreased coronary blood flow. ${ }^{10}$

Lange et al. ${ }^{11}$ conducted the only prospective human trial to examine the effect of beta-selective blockade on coronary artery hemodynamics in the setting of cocaine. This article is the primary source cited throughout the medical literature when discussing beta-blocker usage in the setting of cocaine chest pain and ACS. In this study, ten human subjects received intracoronary propranolol following intranasal cocaine administration. Similar to the canine study, Lange et al., found a significant increase in coronary vascular resistance, and a decrease in coronary sinus blood flow. ${ }^{11}$

The only other human study that has looked at coronary blood flow following beta blockade in the setting of cocaine intoxication was published by Boehrer et al., ${ }^{12}$ three years after Lange's article. This study looked at coronary artery area following intranasal cocaine and then administration of labetalol, a combination alpha and beta-blocker. Contrary to Lange's findings, the nine patients that received cocaine followed by labetalol did not show a decrease in coronary artery area. ${ }^{12}$

Thus, these three studies comprise the entirety of the literature evaluating this topic, and form the basis for the current national guidelines. With only two prospective human studies involving a total of 19 patients and contradictory results, it is not possible 
to draw a valid evidence-based conclusion on the cardiovascular effects of beta blockade in the setting of cocaine intoxication. More data regarding coronary perfusion as well as actual patient outcomes are needed before firm recommendations can be drawn regarding beta-blocker usage in this setting.

\section{Beta-blockers Effect on Systemic Hemodynamic Pa- rameters and Outcome}

Though not specifically mentioned as a reason for beta-blocker avoidance in the ACC/AHA guidelines, beta-blockers in the setting of recent cocaine use have long been hypothesized to worsen systemic hypertension and tachycardia, and possibly precipitate myocardial ischemia due to increased cardiac oxygen demand. The mechanism is attributed to unopposed alphareceptor stimulation leading to worsening systemic vasoconstriction. ${ }^{13}$ In this area, the evidence is equally inconsistent, with newer research concluding that beta-blockers may actually be beneficial in patients with cocaine associated chest pain and myocardial ischemia.

Sources that have shown worsening of hypertension, tachycardia, and cardiovascular outcomes secondary to beta blockade are scarce and have been confined to case reports, animal studies, and small retrospective case studies. ${ }^{14,15,16}$ In contrast, data demonstrating improved hemodynamics and cardiovascular outcomes from beta blockade following cocaine intoxication is derived from more recent research, with larger sample sizes, and in some cases prospective design. ${ }^{12,17,18,19,20,21}$

One of the most compelling prospective studies favoring the use of beta-blockers in patients with recent cocaine use is a trial conducted by Hoskins et al. ${ }^{20}$ in which 90 patients with ACS and cocaine positive urine drug screen were randomized to labetalol or diltiazem. Both subject groups experienced similar and statistically significant decreases in blood pressure and heart rate, with no adverse outcomes occurring in any of the 60 patients that received combined alpha and beta-blocker therapy. ${ }^{20}$

Rangel et al. ${ }^{19}$ conducted a retrospective cohort review looking at patients presenting with chest pain who were also found to have a cocaine positive urine drug screen. 331 patients were included, with $151(46 \%)$ receiving beta-blocker therapy $(85 \%$ received metoprolol). ${ }^{19}$ Patients that received a beta-blocker in the emergency room (ER) had a significantly greater decrease in systolic blood pressure, and there was no difference in the rate of adverse outcomes, peak troponin levels, or EKG changes when compared with patients that did not receive beta-blocker therapy. ${ }^{19}$ There was also a post-hospitalization decreased mortality rate observed in the patients that received beta-blockers in the ER. ${ }^{19}$

Recent data appear to show that selective beta-blockade as well as combination alpha and beta-blockade in the setting of cocaine intoxication do not increase the rate of negative outcomes. In fact most cases have shown improved hemodynamics and more favorable cardiovascular outcomes.

\section{Conclusion}

Given the strong evidence supporting the morbidity and mortality benefits of beta-blockers in patients undergoing ACS, and the weak and contradictory evidence opposing them in the setting of cocaine use it is necessary that steps are taken to better understand the issue. The majority of the recent data does not show any adverse cardiovascular outcomes, and the older data are equivocal at best.

We are possibly withholding beneficial treatment to patients based on unclear and outdated evidence. It is necessary to further examine the current treatment of cocaine related chest pain with prospective, randomized human trials to best determine what is optimal for patient outcomes.

\section{References}

1. 2008 National Survey on Drug Use and Health. US Department of Health and Human Services. www.oas.samhsa.gov

2. Substance Abuse and Mental Health Service Administration, Office of Applied Studies. Drug Abuse Warning Network, 2007: National Estimates of Drug-Related Emergency Department Visits. Rockville, MD, 2010.

3. Brody SL, Slovis CM, Wrenn KD. Cocaine-related Medical Problems: consecutive series of 233 patients. Am J Med. 1990; 88:325-331.

4. Hollander JE, Hoffman RS, Gennis P, et al. Prospective multicenter evaluation of cocaine-associated chest pain. Cocaine Associated Chest Pain (COCHPA) Study Group. Acad Emerg Med. 1994; 1(4): 330-9.

5. Antman EM, Anbe DT, Armstrong PW, et. al ACC/AHA Guidelines for the Management of Patients With ST-Elevation Myocardial Infarction-Executive Summary: A Report of the American College of Cardiology/American Heart Association Task Force on Practice Guidelines (Writing Committee to Revise the 1999 Guidelines for the Management of Patients With Acute Myocardial Infarction). Circulation. 2004; 110: 588-636.

6. Braunwald E, Antman EM, Beasley JW, et al. ACC/AHA Guidelines for the Management of Patients With Unstable Angina and Non-ST-Segment Elevation Myocardial Infarction: Executive Summary and Recommendations: A Report of the American College of Cardiology/American Heart Association Task Force on Practice Guidelines (Committee on the Management of Patients With Unstable Angina). Circulation. 2004; 102: 1193-1209.

7. Randomised trial of intravenous atenolol among 16027 cases of suspected acute myocardial infarction: ISIS-1. First International Study of Infarct Survival Collaborative Group. Lancet. 1986; 2:57-66.

8. The MIAMI Trial Research Group. Metoprolol in acute myocardial infarction: patient population. Am J Cardiol. 1985; 56:10G-14G.

9. Kloner RA, Hale S. Unraveling the complex effects of cocaine on the heart. Circulation. 1993; 87(3): 1046-7.

10. Shannon RP, Stambler BS, Komamura K, et al. Cholinergic modulation of the coronary vasoconstriction induced by cocaine in conscious dogs. Circulation. 1993; 87(3): 939-49.

11. Lange RA, Cigarroa RG, Flores ED, et al. Potentiation of cocaine, induced coronary vasoconstriction by beta adrenergic blockade. Ann Intern Med. 1990; 112:897-903.

12. Boehrer JD, Moliterno DJ, Willard JE, et al. Influence of labetalol on cocaineinduced coronary vasoconstriction in humans. Am J Med. 1993; 94(6): 608-10.

13. McCord J, Jneid H, Hollander JE, et al. Management of cocaine-associated chest pain and myocardial infarction: a scientific statement from the American Heart Association Acute Cardiac Care Committee of the Council on Clinical Cardiology. Circulation. 2008; 8:117(14): 1897-907. 
14. Ramoska E, Sacchetti AD. Propranolol-induced hypertension in treatment of cocaine intoxication. Ann Emerg Med. 1985; 14(11):1112-3.

15. Smith M, Garner D, Niemann JT. Pharmacologic interventions after an LD50 cocaine insult in a chronically instrumented rat model: are beta-blockers contraindicated? Ann Emerg Med. 1991; 20(7): 768-71.

16. Sand IC, Brody SL, Wrenn KD, et al. Experience with esmolol for the treatment of cocaine-associated cardiovascular complications. Am J Emerg Med. 1991; 9(2): 161-3.

17. Rappolt RT, Gay G, Inaba DS, et al. Propranolol in cocaine toxicity. Lancet. 1976 $18 ; 2$ (7986):640-1.

18. Catravas JD, Waters IW. Acute cocaine intoxication in the conscious dog: studies on the mechanism of lethality. J Pharmacol Exp Ther. 1981; 217(2): 350-6.
19. Rangel C, Shu RG, Lazar LD, et al. Beta-blockers for chest pain associated with recent cocaine use. Arch Intern Med. 2010; 170(10): 874-9.

20. Hoskins MH, Leleiko RM, Ramos JJ, et al. Effects of labetalol on hemodynamic parameters and soluble biomarkers of inflammation in acute coronary syndrome in patients with active cocaine use. J Cardiovasc Pharmacol Ther 2010; 15(1): 47-52

21. Dattilo PB, Hailpern SM, Fearon K, et al. Beta-blockers are associated with reduced risk of myocardial infarction after cocaine use. Ann Emerg Med. 2008; 51(2): 117-25.

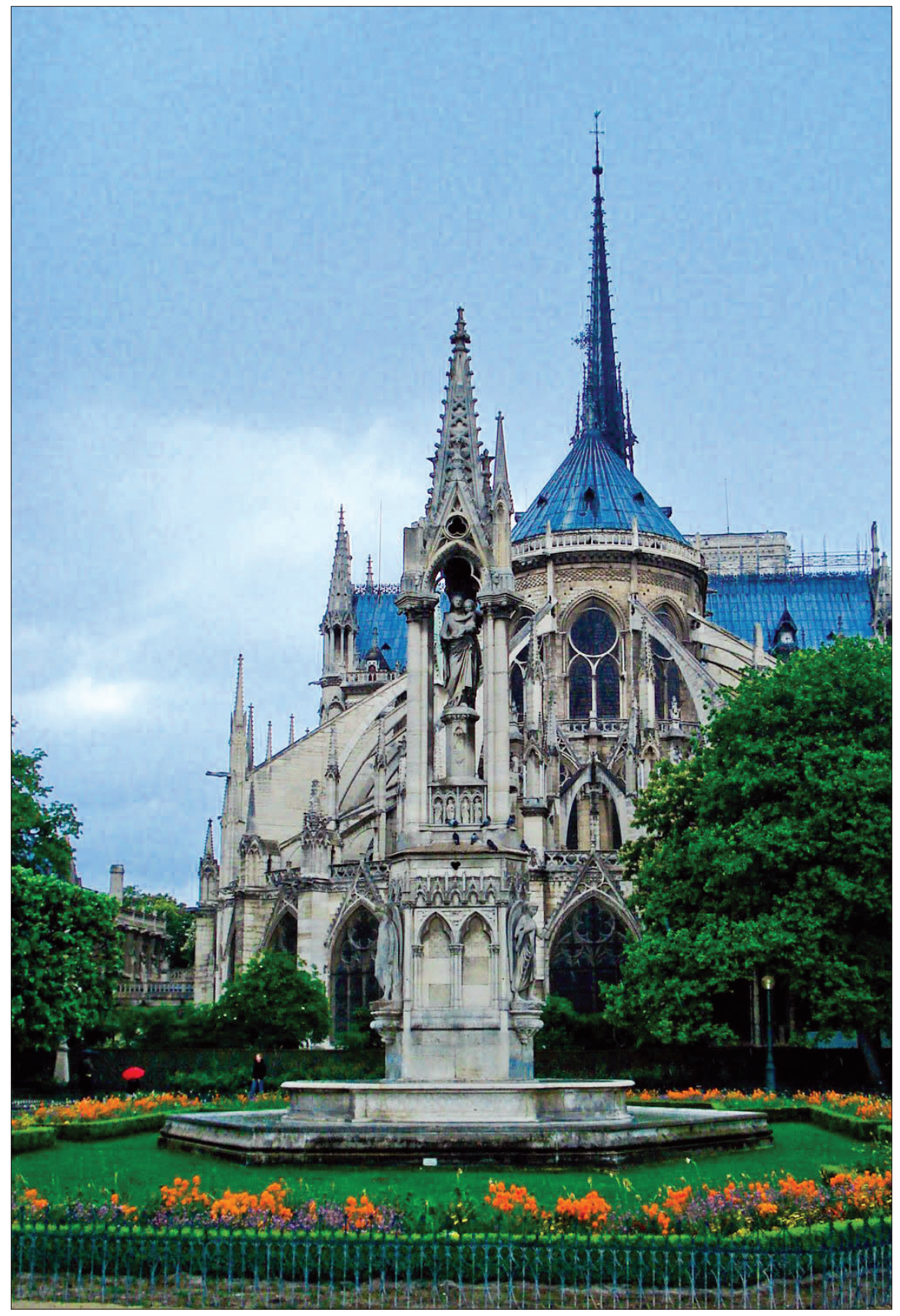

"Back of Big Ben"

photograph by Paurush Shah, MD 